\title{
P67 Shows Differential Effects in Proteolysis and Maturation of Cell Cycle Regulatory Proteins, Cyclins and Cdks
}

\author{
Samit K. Datta, Bansidhar Datta* \\ Department of Chemistry and Biochemistry, Kent State University, Kent, OH 44240, USA \\ "bdatta@kent.edu
}

\begin{abstract}
The level of p67 gradually increases when mouse C2C12 myoblasts differentiate into myotubes. During this differentiation, cycling myoblasts permanently withdraw from cell cycle. Largely, the cyclin/Cdk complexes drive eukaryotic cell cycle. To investigate whether p67 has any role(s) while C2C12 myoblasts are withdrawing from the cell cycle, we examined the levels of cyclins and Cdks in these myoblasts constitutively expressing rat p67 and its mutants D251A, H231E, D6/2, and K1K2. We found that p67 is involved in lowering the overall concentration of cyclin D1/Cdk4 and cyclin D1/Cdk6 complexes and thus inhibits the cell cycle at the $G_{1}$-phase. It is also involved in possible proteolytic processing of pro-forms of cyclins $E, A$, and BI but not cyclin D1 to maturation. P67 may be involved in lowering the level of Cdk2 possibly by proteolytic degradation and increasing the level of Cdk1 by an unknown mechanism. P67 is involved in the increased level of cyclin A possibly to show positive outcome during DNA synthesis. It also showed strong effects on degradation of cyclin $B 1$ possibly due to their direct or indirect binding - indicating p67's involvement in the inhibition of $G_{2}$-phase and $G_{2} / M$-phase transition. We also observed that when $p 67$ 's auto-proteolysis activity was increased due to the change of H231 residue to E231, this mutant showed dominating effect over endogenous p67. Altogether, our data indicate that increased level of p67 during myoblasts' differentiation into myotube is possibly to inhibit cell cycle at the $G_{1}$-phase, $G_{1} / S$-phase transition, $G_{2}$-phase, and $G_{2} / M$-phase transition.
\end{abstract}

Keywords: cellular glycoprotein p67 that binds to eIF2 and ERK1/2, cell cycle, cyclins and cdks, fumagillin, p67 mutants, pro-forms of cyclins, proteolysis

Abbreviations used: p67, a 67 kDa glycoprotein that binds to both eukaryotic initiation factor 2 (eIF2) and extracellular signal-regulated kinases 1 \& 2 (ERK1/2); D251A and H231E, p67 point mutants, which had alanine substitution for aspartic acid at 251 amino acid residue and glutamic acid substitution at 231 histidine amino acid respectively; D6/2, a p67 block mutant, where a stretch of acidic amino acid residues at the $N$ terminus has been replaced with uncharged amino acids; K1K2, a p67 block mutant, where both of its lysine/arginine-rich stretches of amino acid residues at the $\mathrm{N}$-terminus have been replaced with uncharged amino acids; CDKs, cyclin-dependent kinases; MAP, mitogen-activated protein; Ab, antibody; IB, immunoblotting; $I P$, immunoprecipitation; and $\operatorname{Ig} G(H) \& \operatorname{Ig} G(L)$ for immunoglobulin heavy chain and light chain respectively.

\section{INTRODUCTION}

The cellular glycoprotein p67 binds to both eukaryotic initiation factor 2 (eIF2), which regulates rates of global protein synthesis (1-3) and extracellular signal-regulated kinases (ERK1/2) (4), which modulates cell growth and proliferation, differentiation, and apoptosis (5). P67's binding to these proteins suppresses the levels of phosphorylation of the smallest $\alpha$-subunit of eIF2 and ERK1/2 mitogen-activated protein (MAP) kinases both in vitro and ex-vivo cell cultures (1-4). P67 when not bound to either eIF2 or ERK1/2 shows auto-proteolysis activity that generates several peptides including the N-terminal 1-107 amino acid segment (also known as p26), the downstream 108-480 amino acid segment (also known as p52), and possibly others (6). In p26 segment of p67, there are two lysine/arginine - rich domains separated by an acidic residue-rich sequences (1, 6-7). In the p52 segment of p67, there are five conserved amino acid residues - D251, D262, H331, E364, and E459, which together fold to create a shallow pocket (8), where p26 segment can snuggly "fit-in" bringing R108 residue closer to the catalytic site $\mathrm{H} 231$ for auto-proteolysis (1, 6-7). Nonetheless, the p52 segment of p67 alone shows auto-proteolysis activity at least in vitro (6) and possibly in vivo. The auto-proteolysis activity of p67 is however inhibited by fumagillin, an anti-angiogenic drug isolated from fungi (7-8) and this leads to the accumulation of the protein inside the cells due to the decrease of its turnover rate (9). Increased levels of p67 inside cells binds to ERK1/2 MAP kinases and inhibits their activation and activity in vitro and ex-vivo cell cultures (9). 
Activation and activity of ERK1/2 MAP kinases is essential for the progression of cell cycle (5). Mammalian cell cycle is a highly regulated cellular process and any deregulation of this step of cell division will lead to uncontrolled cell growth or in situ tumor formation (10-12). The different phases of cell cycle are properly initiated, maintained, progressed to the next phase, and regulated in each phase of the cycle by a group of complexes consist of cyclins and cyclin-dependent kinases (cdks). For example, D-group of cyclins (cyclin D1, D2, and D3) play important roles during growth phase $\left(\mathrm{G}_{1}\right.$-phase); cyclin $\mathrm{E}$ is important for the transition of cell cycle from $\mathrm{G}_{1}$-phase to DNA synthesis phase (S-phase); cyclin A is important throughout the $\mathrm{S}$-phase and transition from $\mathrm{S}$-phase to $\mathrm{G}_{2^{-}}$ phase; and cyclin B1 plays important roles in the maintenance of second growth phase $\left(\mathrm{G}_{2}\right.$-phase), where there is a spindle formation to separate $4 \mathrm{n}$ chromosomes to two $2 \mathrm{n}$ chromosomes followed by mitosis (the M-phase). Cyclin B1 is also important for the transition of $\mathrm{G}_{2}$-phase to M-phase and maintenance of early M-phase of the cell cycle. For functionality of the cyclins, Cdk1 makes cyclin/Cdk complexes with cyclin A and cyclin B1, Cdk2 partners with cyclin E and cyclin A, and Cdk4, 6 make complex with D-group of cyclins (13-15). The turnover rates of cdks are much lower than their partners, cyclins, whose degradations basically modulate the transitions from one phase of cell cycle to the next (16). Several proteases either from the proteasomes or in the cytoplasm are involved in the degradation of cyclins and Cdks (16-17).

In this study, we have explored the involvement of p67 in the proteolysis of cyclins and Cdks that regulate the different phases of eukaryotic cell cycle. We specifically examined the levels of both cyclins and cdks in fumagillin-treated and untreated $\mathrm{C} 2 \mathrm{C} 12$ mouse myoblasts constitutively expressing rat p67 and some of its specific mutants. Our data show that substitution of aspartic amino acid at 251 residue with alanine leads to the accumulation of a longer form of cyclins E, A, and B1 but not cyclin D1. Substitution of histidine 231 residue with glutamic acid also leads to the accumulation of a longer form of cyclins $\mathrm{E}$ and A, but not cyclins B1 and D1. In addition, similar results of accumulation of a longer form of cyclins E, A, and B1 but not cyclin D1 are observed when a block mutant of p67 (D6/2), where its N-terminal acidic residue-rich sequences were substituted with neutral amino acid residues, is constitutively expressed in $\mathrm{C} 2 \mathrm{C} 12$ myoblasts. In contrast, the double block mutants (K1K2), where p67's both N-terminal lysine/arginine residue-rich sequences were substituted with neutral amino acid residues, when constitutively expressed in $\mathrm{C} 2 \mathrm{C} 12$ myoblasts showed no accumulation of the longer forms of the above cyclins. Constitutive expression of rat p67 or some of its specific mutants in the $\mathrm{C} 2 \mathrm{C} 12$ myoblasts showed some degradation of Cdk2 but not Cdk1, Cdk4, or Cdk6 while the level of Cdk1 was rather up-regulated. Dramatic degradation of cyclin B1 in mouse cells constitutively expressing p67 may be due to their binding either directly or indirectly. Together, our data suggest that p67 is involved in the degradation of certain cyclins and Cdks and thus modulates the progression of cell cycle.

\section{Materials ANd Methods}

All chemicals used in this study were obtained from Sigma Chemicals (St. Louis, MO), Merck (Darmstadt, Germany), ICN Biomedicals, Inc. (Aurora, Ohio), Fisher Chemicals (New Jersey), or GIBCO-BRL (Rockville, MD). All enzymes used in this study were purchased from New England Biolabs (Beverly, MA). Molecular mass markers were purchased from BioRad.

\subsection{Generation and Sequencing of Various P67 Mutants}

The rat p67 and its mutants like D251A, D6/2, K1K2, and H231E were generated by site-directed mutagenesis, sequenced to verify mutations, and subcloned into EGFP expression vector to obtain inframe-fusions following the procedures as described (18). The expressions and sizes of the EGFPfusion proteins were examined on Western blots using monoclonal antibody specific to EGFP as described (18).

\subsection{Antibodies}

Monoclonal antibodies specific to cyclin A (Sc-751), cyclin B1 (Sc-245), cyclin D1 (Sc-20044), cyclin E (Sc-247 \& Sc-481), Cdk1 (Sc-54), Cdk2 (Sc-53220), Cdk4 (Sc-56277), and Cdk6 (Sc-7961) were purchased from Santa Cruz Biotechnology. A monoclonal antibody specific to p53 (Ab-5) was from Calbiochem. Monoclonal antibody specific to $\alpha$ Actin was obtained from Sigma Chemicals and polyclonal antibodies specific to rabbit p67 were raised and characterized following the procedures as described (2). 


\subsection{Cell Culture and Generation of Stable Cell Lines}

$\mathrm{C} 2 \mathrm{C} 12$ mouse myoblasts (ATCC) cultures were maintained in growth medium as described $(9,19$ 21). 50-60\% confluent cultures of $\mathrm{C} 2 \mathrm{C} 12$ cells were transfected with the pEGFP-C 3 vector (Clontech, Palo Alto, CA) for the expression of EGFP alone or its in-frame fusion proteins with either rat p67 or its different mutants. Stable cell lines were generated following the procedures as described (19). In brief, the individual pEGFP-C3 plasmid containing rat p67 or its mutants was mixed with Lipofectamine, DNA precipitates were added to $\mathrm{C} 2 \mathrm{C} 12$ cells, and kept for $6 \mathrm{~h}$ inside the $\mathrm{CO}_{2}$ incubator. The medium was removed, fresh growth medium was added to the transfected cells, and cells were grown for additional $48 \mathrm{~h}$ in $\mathrm{CO}_{2}$ incubator. Subsequently, transfected cells were harvested and distributed in ten cell culture dishes and allowed to grow in the presence of $1.2 \mathrm{mg} / \mathrm{mL}$ of G418. The fresh medium containing G418 was replaced every five days. Approximately two to three weeks later, about 100-200 well-defined colonies from ten cell culture dishes were pooled and allowed to grow in the presence of $0.6 \mathrm{mg} / \mathrm{mL}$ of G418. The pooled mixed colonies of transfected cells were used for further experiments.

\subsection{Preparation of Total Cell Extracts, and Western Blottings Fumagillin Treatment of Cells from Various Lines}

Fumagillin (Sigma Chemical, St. Louis, MO) was dissolved in DMSO, and if so indicated, C2C12 myoblasts at $60 \%$ confluence were treated with $12 \mu \mathrm{M}$ fumagillin for $36 \mathrm{~h}$. Procedures for the preparation of cell lysates and Western blots were essentially the same as described (19). Protein bands in Western blots were scanned and their intensities were quantitatively measured by NIH Image 162 software program.

\subsection{Immunoprecipitation Assays}

For immunoprecipitation assays, 1-5 $\mu \mathrm{g}$ of either monoclonal or polyclonal antibodies were bound to protein $\mathrm{A}$ - agarose, free antibodies were removed by centrifugation and 300-500 $\mu \mathrm{g}$ of total protein samples were added to the antibody-bound protein $\mathrm{A}$ - agarose beads and mixed by gentle shaking at $4{ }^{\circ} \mathrm{C}$ for $12-16 \mathrm{~h}$. The unbound proteins were removed by brief centrifugation and the beads were washed extensively with washing buffer. Subsequently, beads were resuspended with 5xSDS-PAGE loading buffer, the mixtures were boiled for $10 \mathrm{~min}$, microfused briefly, and the clear supernatants were loaded on $15 \%$ SDS-PAGE followed by Western blot analysis.

\section{Results}

\subsection{The D251 Amino Acid Residue and the N-Terminal Acidic Domain of P67 may be Involved in the Maturation of Cyclin E, Cyclin A, and Cyclin B1 but not Cyclin D1 in C2C12 Mouse Myoblasts}

Stable cell lines of $\mathrm{C} 2 \mathrm{C} 12$ mouse myoblasts expressing EGFP or its in-frame fusions of rat p67 and its mutants, D251A, D6/2, and K1K2 were maintained in growth medium containing G418. These cell lines expressed almost equal amounts of either EGFP or its fusion proteins of rat p67 or its mutants (data not shown). Description of p67-mutants used in this study along with several other p67-mutants is reported (18). Cell lysates from these cell lines either treated or untreated with fumagilin were analyzed for the levels of cyclin E, cyclin A, and Cdk2 on Western blots (Fig. 1). We detected basal level of cyclin E in cells constitutively expressing EGFP, EGFP-p67, and EGFP-K1K2 (Fig. 1A, lanes 1-4 and 9-10). Untreated cells expressing D251 and D6/2 mutants however showed some accumulation of a slower migrating cyclin E form (we named it "pro" cyclin E) and this accumulation is reduced significantly when cells were treated with fumagillin (compare lanes $5 \& 7$ with lanes $6 \&$ 8 in Figure 1A). Fumagillin treatment of $\mathrm{C} 2 \mathrm{C} 12$ muscle cells leads to the accumulation of endogenous p67 due to the reduction of its turnover rate (9). In case of cyclin A level in these above mentioned cell lines, we got mixed results (Fig. 1B). For example, fumagillin treatment of control cells increased the levels of cyclin A by at least 3-4 folds (Fig. 1B, compare lane 1 with lane 2). Cells expressing rat p67 has at least 6-7 folds of cyclin A level as compared to control EGFP-expressing cells either treated or untreated with fumagillin (Fig. 1B lanes 3-4). Cyclin A level in untreated cells expressing D251 mutant is $\sim 10$-fold higher than control EGFP-expressing cells (Fig.1B, compare lane 5 with lane 1). This high level however decreased to 3-4 folds when cells were treated with fumagillin (Fig. 1B, 
compare lane 6 with lane 1). Stable untreated C2C12 cells expressing D6/2 mutant had $\sim 5$-fold increase in cyclin A level as compared to control EGFP-expressing cells and this level reduced to 2fold to near control level when cells were treated with fumagillin (Fig. 1B, compare lane 8 with lane 1). Cells expressing K1K2 mutant show near control level of cyclin A and this level did not change when cells were treated with fumagillin (Fig. 1B, lanes 9-10). Most importantly, the slower migrating cyclin A (we named it "pro" cyclin A) was accumulated significantly in D251A-expressing and D6/2expressing cells (Fig. 1B, lanes $5 \& 7$ ). This form of cyclin A however disappeared when cells were treated with fumagillin (Fig. 1B, lanes $6 \& 8$ ). The levels of Cdk 2 decreased $\sim 2-3$ folds consistently in the above stable $\mathrm{C} 2 \mathrm{C} 12$ cells when treated with fumagillin as compare to their untreated counterparts (Fig. 1C, compare lanes 1, 3, 5, 7, and 9 with lanes 2, 4, 6, 8, and 10 respectively). In addition, cells expressing either rat p67 or its $\mathrm{K} 1 \mathrm{~K} 2$ mutant showed 2-3 fold decrease in cyclin A as compared to control cells expressing EGFP alone (Fig. 1C, compare lanes $3 \& 9$ with lane 1). Altogether, our data suggest that the conserved aspartic acid at 251 location and the N-terminal acidic residue-rich domain of p67 are involved in the maturation of slower migrating "pro" cyclin A and "pro" cyclin E in $\mathrm{C} 2 \mathrm{C} 12$ myoblasts. The change in levels of Cdk2 in fumagillin treated cells may be due to higher levels of endogenous p67 and the exogenously expressed D251A, D6/2, or K1K2 mutants do not interfere with p67's function.

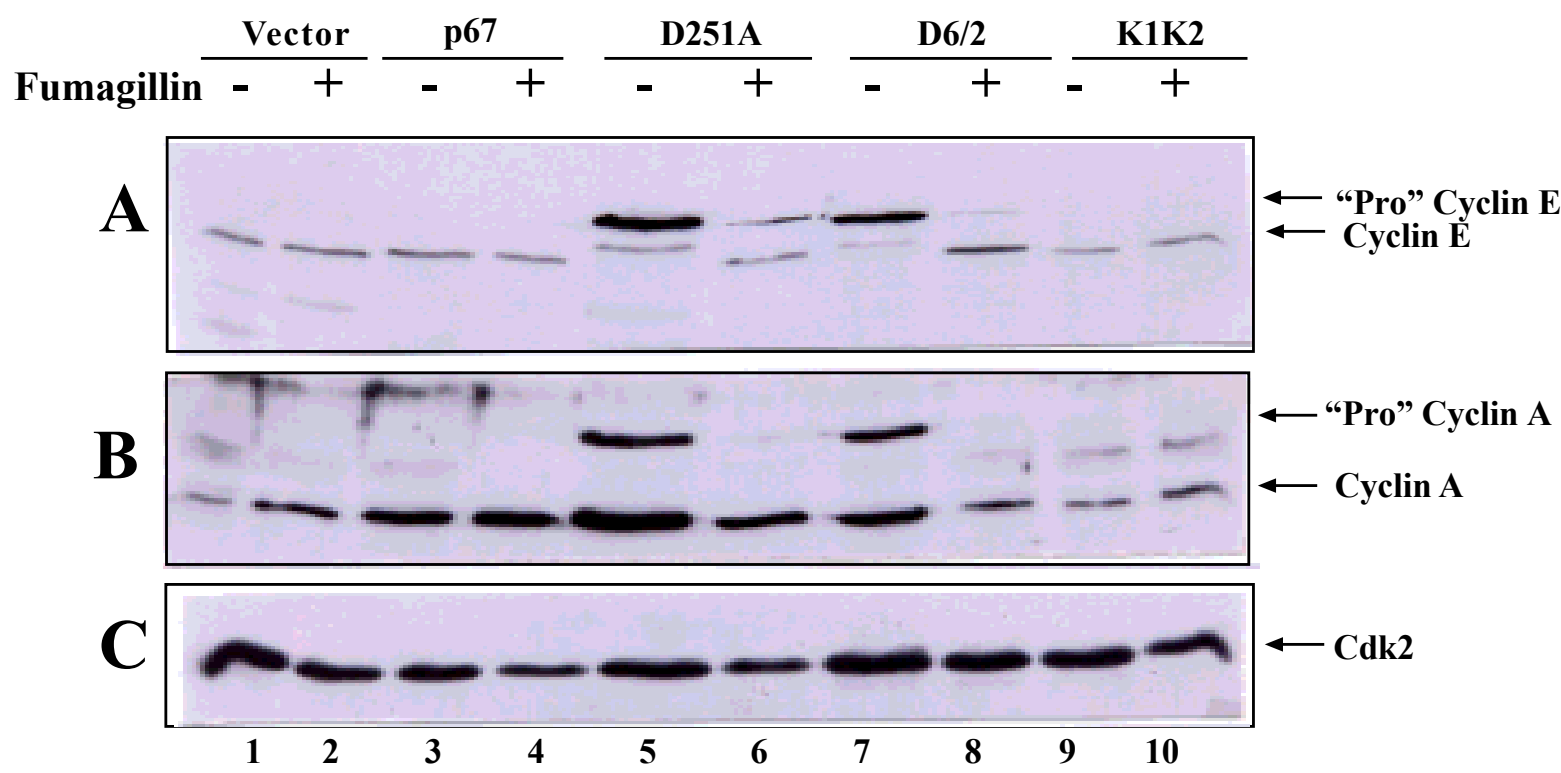

Fig1. Levels of cyclin E, cyclin A, and Cdk2 in C2C12 myoblasts constitutively expressing EGFP-expression vector, rat 667 and its mutants D251A, D6/2, and K1K2. Mouse C2C12 myoblasts constitutively expressing the EGFP vector or its in-frame fusions with wild type rat p67, the D251A, D6/2 and K1K2 mutants were treated for $36 \mathrm{~h}$ with $12 \mu \mathrm{M}$ fumagillin. Cell lysates prepared from both fumagillin-treated and untreated cells were analyzed on 15\% SDS-PAGE followed by Western blots with antibodies specific to cyclin E (panel A), cyclin A (panel B), and Cdk2 (panel C). The slower migrating proteins that were detected by cyclin $E$ and cyclin $A$ antibodies are designated as the "pro" forms of these cyclins

Next, we examined the levels of cyclin D1 and its partner Cdk4 on Western blots in cell extracts used in our experiments mentioned in Figure 1. Our results show that there is a 60-70\% decrease in cyclin D1 level in fumagillin-treated cells expressing EGFP, EGFP-p67, and EGFP-K1K2 as compared to their untreated cell extracts (Fig. 2A, compare lanes 2, 4, and 10 with lanes 1, 3, and 9). Cell expressing EGFP-D251A and EGFP-D6/2 mutants however did not show any significant change in their cyclin D1 levels whether cells were treated with fumagillin or not (Fig. 2A, see lanes 4-8) although, the levels of cyclin D1 in EGFP-D6/2 expressing cells were 3-4 fold lower as compared to control untreated cells (Fig. 2A, compare lanes 7-8 with lane 1). Levels of Cdk4 in the above mentioned cell lines changed slightly, except in EGFP-D6/2 expressing cells, which showed 3-4 fold lower than control EGFP-expressing cells (Fig. 2B, compare lanes 7-8 with lane 1). Altogether, our results indicate that endogenous p67 has some effects on possible degradation of cyclin D1/Cdk4 complex in general. Nonetheless, unlike cyclin E and cyclin A, we have not detected any "pro" form of cyciln D1 in our experimental cell lines used in this study. 
P67 Shows Differential Effects in Proteolysis and Maturation of Cell Cycle Regulatory Proteins, Cyclins and Cdks

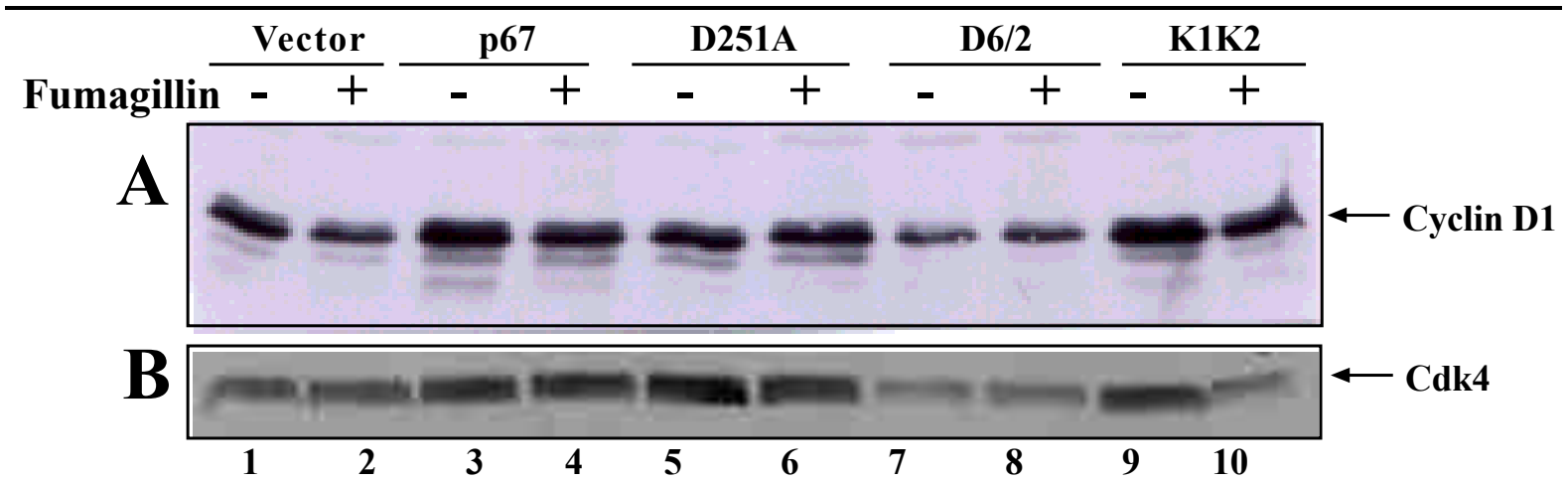

Fig2. Levels of cyclin D1 and Cdk4 in C2C12 myoblasts constitutively expressing rat p67 and some of its mutants. Similar experiments in cell extracts from different cell lines as mentioned in legend to Figure 1 were performed except the analyses were done for the levels of cyclin DI and Cdk4 using antibodies specific to these proteins.

Our examination of the levels of cyclin B1 in the above-mentioned cell lines showed some surprising results (Fig. 3). The full-length cyclin B1 was not detected in control cells expressing EGFP or cells expressing EGFP-K1K2 mutant either treated or untreated with fumagillin (Fig. 3A, see lanes 1-2 \& 9-10). Cyclin B1 was extensively degraded in rat p67-expressing cells either treated or untreated with fumagillin (Fig. 3A, lanes 3-4) and similar to pro-cyclin E and pro-cyclin A, the "pro" form of cyclin B1 is detected in both D251A- and D6/2-mutant expressing cells (Fig. 3A, lanes 5-8). The level of $\mathrm{Cdk} 1$, the partner of cyclin A and cyclin B1, remains basically unaffected by the fumagillin treatment of control EGFP-expressing cells (Fig. 3B, lanes 1-2). Its level in rat p67-expressing cells remains very similar to control cells but it increased to 6-7 folds when these cells were treated with fumagillin (Fig. 3B, compare lane 1-3 with lane 4). In D251A-expressing cells, the level of Cdk1 is about 3-4 fold higher and it went to 4-5 fold higher when treated with fumagillin (Fig. 3B, compare lanes 5 \& 6 with lane 1). The D6/2-expressing cells showed most dramatic increase in Cdk1 level up to 7-8 fold as compared to control cells (Fig. 3B compare lane 7 with lane 1) and its level dropped to 5-6 fold when treated with fumagillin (Fig. 3B lane 8). On the other hand, Cdk1 level was higher by 3-4 fold and it went up to 5-6 fold higher when K1K2 mutant-expressing cells were treated with fumagillin (Fig. 3B, compare lanes 9-10 with lane 1). These results therefore indicate that endogenous p67 may be involved in the up-regulated expression of Cdk1 and p67's conserved amino acid D251, its acidic residue-rich sequences and both $\mathrm{N}$-terminal stretches of lysine/arginine residue-rich sequences are involved in the down regulation of Cdk1.

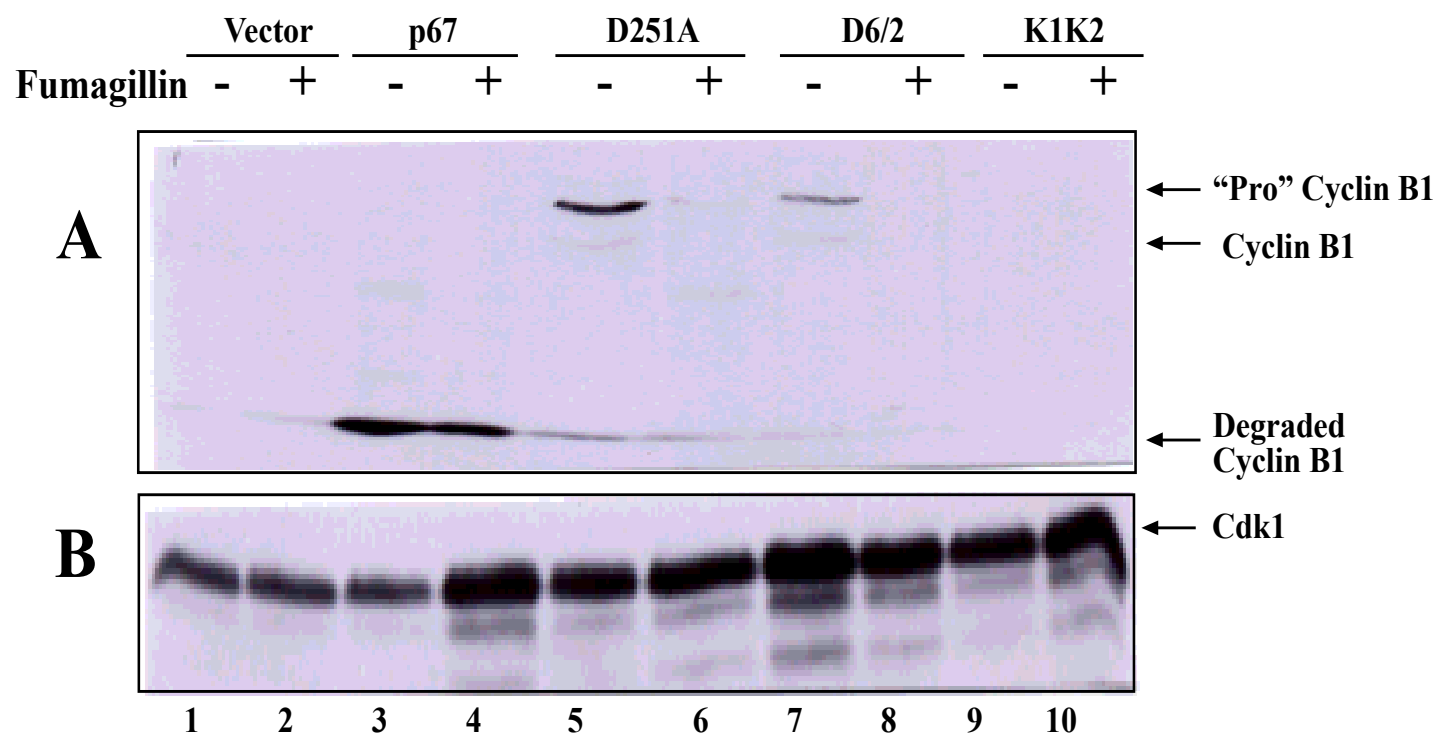

Fig3. Levels of cyclin $B 1$ and Cdk1 in C2C12 myoblasts constitutively expressing rat p67 and some of its mutants. Similar experiments were performed as mentioned in legend to Figure 1 except the analyses were done for the levels of cyclin BI and CdkI using antibodies specific to these proteins 


\subsection{P67 Binds to Cyclin B1 and Cdk1}

Our results from the analysis of the levels of cyclin B1 in the above-mentioned cell lines used in experiments in Figure 3 raised question whether p67 and cyclin B1 interact. We used three cell lines (i) control cells expressing EGFP, (ii) rat p67-expressing cells and (iii) cell expressing D251A mutant. Equal amounts of cell extracts from these lines were used for the immunoprecipitations by Cdk1 monoclonal antibody (Fig. 4A), p67 polyclonal antibodies (Fig. 4B \& 4D), cyclin B1 monoclonal antibody (Fig. 4C), and an unrelated p53 monoclonal antibody (Fig. 4E). After extensive washing, the immunoprecipitates were analyzed on immunoblots using antibodies for p67 (Fig 4A, C, and E), Cdk1 (Fig. 4B), and cyclin B1 (Fig. 4D). 10\% equivalent amounts of total cell extracts used for immunoprecipitations, were analyzed for the levels of Cdk1 (Fig. 4A, lower panel), p67 (Fig. 4B \& 4D, lower panels), cyclin B1 (Fig. 4C, lower panel), and p53 (Fig. 4E), lower panel). Together, our immunoprecipitation experiments show that p67 binds to both cyclin B1 and its partner Cdk1 and its D251A mutant does not show strong binding to cyclin B1 although, it binds to Cdk1.

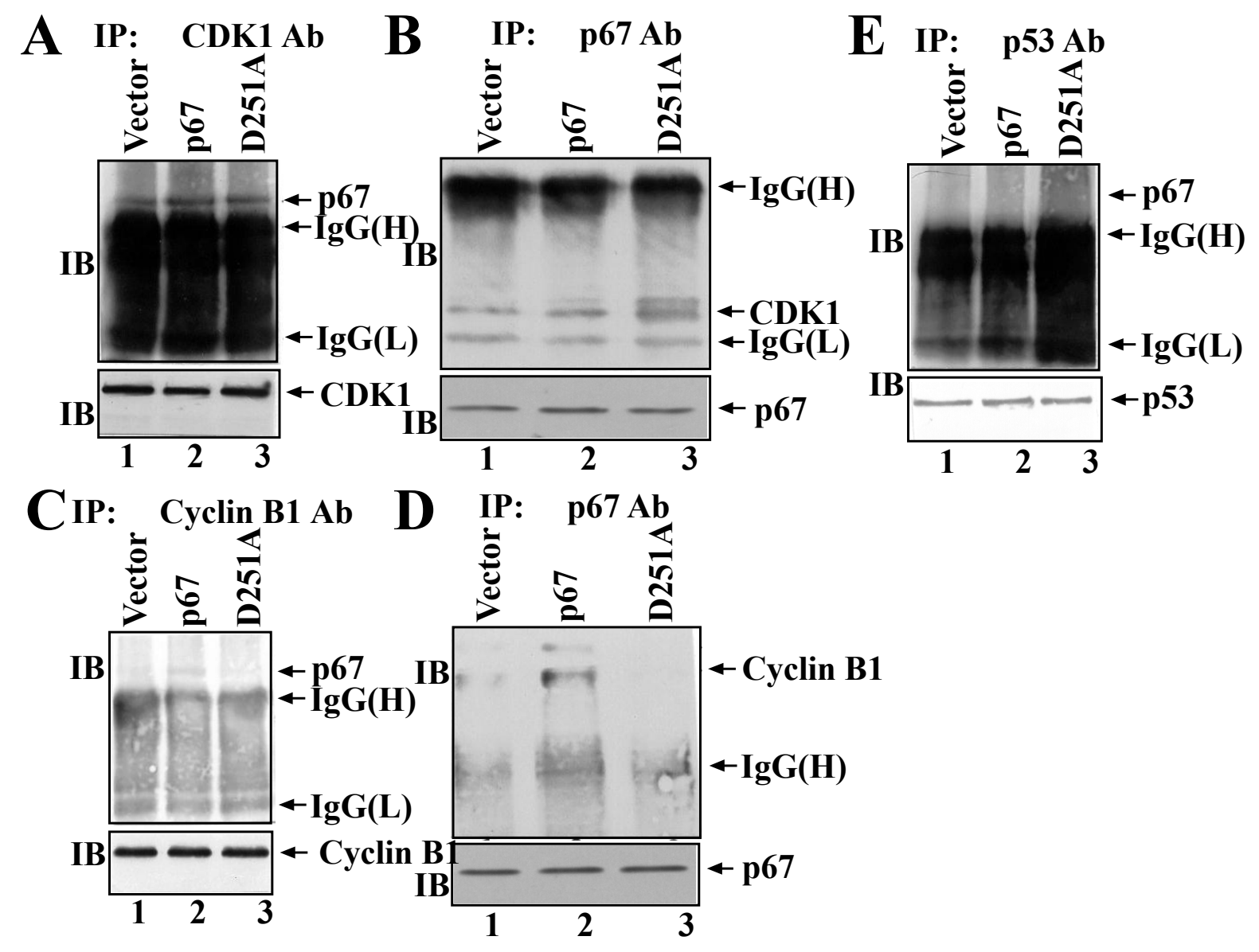

Fig4. Immuno-precipitation assays for testing the interactions between p67, cyclin B1, and Cdk1

Immuno-precipitation experiments were performed in total cell lysates obtained from rat tumor hepatoma (KRC-7) cells constitutively expressing EGFP vector or its in frame-fusions of rat p67 and D251A mutant using monoclonal antibody specific to Cdk1 (A), polyclonal antibodies specific to p67 (B \& D), monoclonal antibody specific to cyclin B1 (C), and monoclonal antibody specific to p53 (E). The corresponding immuno-precipitates were then analyzed on Western blots using antibodies specific to p67 (A, C, and E, upper panels), Cdk1 (B, upper panel), and cyclin B1 (D, upper panel). $10 \%$ of the total cell extracts were also analyzed on immuno-blots for the input levels of Cdk1 (A, lower panel), p67 (B \& D, lower panels), cyclin B1 (C, lower panel), and p53 (E, lower panel). IP, immuno-precipitation; IB, immuno-blotting; $\operatorname{IgG}(\mathrm{H})$, immunoglobulin heavy chain; and $\operatorname{IgG}(\mathrm{L})$, immunoglobulin light chain.

\subsection{The H231 Amino Acid Residue of P67 may be Involved in Maturation of Cyclin $E$ and Cyclin A but not Cyclin B1 and Cyclin D1 in C2C12 Mouse Myoblasts}

The H231 amino acid residue of p67 has been shown to be the fumagillin binding site (8). Fumagillin binding to p67 inhibits its auto-proteolysis activity and protein accumulates inside the cells (9). We 

and Cdks

therefore performed similar analysis for the levels of cyclin E, cyclin A, cyclin B1, cyclin D1, and their partners like $\mathrm{Cdk} 2$ and $\mathrm{Cdk} 6$ in $\mathrm{C} 2 \mathrm{C} 12$ myoblasts constitutively expressing EGFP and EGFPfusions of rat p67 and its mutant H231E (Figs. 5-6). Our Western blotting experiments with both cyclin E and cyclin A detected the slower migrating "pro" forms of these cyclins in cells expressing H231E mutant and these "pro"-forms however did not disappear but rather increased their levels when cells were treated with fumagillin (Fig. 5A \&B, lanes 5-6). In addition, the level of cyclin E was significantly decreased (more than 12 folds) in fumagillin-treated cells expressing H231E mutant (Fig. $5 \mathrm{~A}$, compare lane 6 with lane 1). The levels of Cdk2 remained basically unchanged in these experimental cell lines (Fig. 5C). When compared to control cells, the level of cyclin D1 is about 3-4 fold lower in rat p67-expressing cells either fumagillin-treated or untreated (Fig. 5A, compare lanes 34 with lanes 1-2). Similarly, the level of cyclin D1 is about 3-4 fold lower in H231E-expressing cells and this level even further reduced to 2-3 fold when cells were treated with fumagillin (Fig. 5A, compare lanes 5-6 with lane 1). The level of Cdk6, the partner of cyclin D1, decreased slightly in the above-mentioned cell lines treated with fumagillin (Fig. 5B, lanes 1-6). We performed similar experiments to measure the levels of cyclin B1 and did not detect any "pro" form of this cyclin (data not shown). Altogether, our data show that histidine 231 residue of p67 may be involved in the maturation of cyclin E and cyclin A but not cyclin B1 and cyclin D1. In addition, this residue may also be involved in the degradation of cyclin $\mathrm{E}$ and cyclin $\mathrm{A}$.

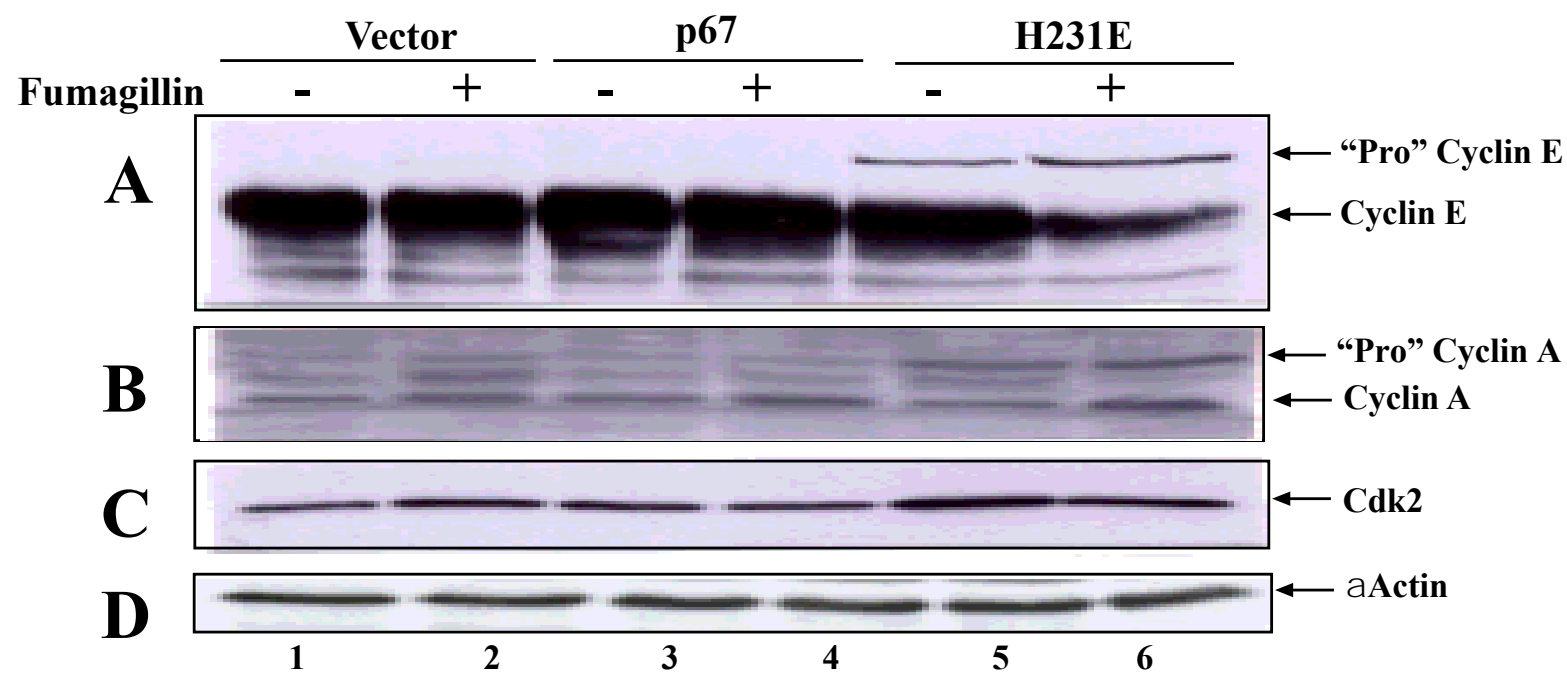

Fig5. Levels of cyclin E, cyclin A, and Cdk2 in C2C12 myoblasts constitutively expressing EGFP vector, rat p67 and its mutant H231E. Mouse C2C12 myoblasts constitutively expressing the EGFP vector or its in-frame fusions with wild type rat 067 from and its mutant H231E were treated for $36 \mathrm{~h}$ with $12 \mu M$ fumagillin. Cell lysates prepared from both fumagillin-treated and untreated cells were analyzed on 15\% SDS-PAGE followed by Western blots with antibodies specific to cyclin E (panel A), cyclin A (panel B), cdk2 (panel C), and $\alpha$ Actin (panel D). The slower migrating proteins that were detected by the cyclin $E$ and cyclin $A$ antibodies are designated as the "pro" forms of these cyclins.

\section{DisCUSSION}

The accumulations of the "pro" forms of cyclin E, cyclin A, and cyclin B1 are quite significant in C2C12 cells constitutively expressing the D251A, D6/2, and H231E mutants of rat p67 (Figs. 1, 3, and 5). These "pro" forms however disappeared when cells from these mutant-expressing lines were treated with fumagillin except in $\mathrm{H} 231 \mathrm{E}$ mutant expressing cells. In the later cell line, we rather observed some increase of these "pro" forms during fumagillin treatment. Treatment of $\mathrm{C} 2 \mathrm{C} 12$ myoblasts with fumagillin enhances the stability of p67 inside the cells by decreasing its turnover rate and inhibiting its auto-proteolysis (9). The appearances of the "pro" forms of cyclins E, A, and B1 in D251A-, D6/2-, and H231E-mutant expressing cells followed by their disappearances in fumagillintreated cells except in $\mathrm{H} 231 \mathrm{E}$ expressing cells, clearly indicate that p67 is involved in the maturation of these cyclins and its aspartic acid residue at 251, histidine at 231, and N-terminal acidic residuerich domain are essential for this activity whereas, its $\mathrm{N}$-terminal lysine-rich domains (domain I \& domain II) are not. P67 has the ability to form dimer (18). But, none of D251A, D6/2, and K1K2 mutants showed strong binding with the wild type rat p67 (18). This then indicates the dominating effects of these mutants over endogenous p67, whose level when upregulated by fumagillin treatment 
the effects of these mutants became insignificant as compared to high levels of endogenous p67. We have not examined the binding of p67 with its H231E mutant. But from this study we have some clue that H231E mutant might bind to the endogenous p67 very tightly and therefore endogenous p67 although, its level was upregulated by fumagillin treatment, could not show the processing activity to maturation of cyclin E and cyclin A (Fig. 5).

Our results from this study also show that constitutive expression of rat p67 or its mutants D251A, D6/2, and $\mathrm{K} 1 \mathrm{~K} 2$ in $\mathrm{C} 2 \mathrm{C} 12$ myoblasts had no effect on basal level of cyclin $\mathrm{E}$ (Fig. 1A), significant increase in basal level of cyclin A except K1K2 mutant showed no effect (Fig. 1B), and quite a moderate increase of cyclin D1 except D6/2 mutant, which rather decreased cyclin D1 level (Fig. 2A). Increasing endogenous p67 level by fumagillin treatment showed no effect on basal level of cyclin E, but quite a decreased level of both cyclin A, except the K1K2 mutant that showed no change (Fig. 1B), and cyclin D1, except D6/2 mutant that showed no change either (Fig. 2A). The basal levels of cyclin $\mathrm{E}$ and cyclin A in $\mathrm{H} 231 \mathrm{E}$ mutant-expressing cells were very similar to control cells but, when treated with fumagillin, the cyclin E level went down significantly whereas, cyclin A level slightly increased (Fig. 5A \& 5B, lanes 5-6). Since cyclin $E$ is the regulator of $G_{1} / S$-phase transition and cyclin A regulates DNA synthesis phase, it's possible that p67's histidine 231 residue may be involved in these two phases of the cell cycle indirectly by modulating the activities of unknown proteins. The H231E mutant showed significantly increased auto-proteolysis (6). It is therefore possible that this mutant may also have increased intermolecular proteolysis activity that reduces the cyclin E level significantly (Fig. 5A, lane 6). Increased levels of cyclin A in $\mathrm{C} 2 \mathrm{C} 12$ cells expressing rat p67 and its mutants D251A and D6/2 (Fig. 1A\&B) indicate that p67 may be involved in positive regulation of S-phase of cell cycle. However, p67's ability to lower the level of Cdk2 (see discussion below), the partner of cyclin E, also indicates its role beyond cell cycle regulation.
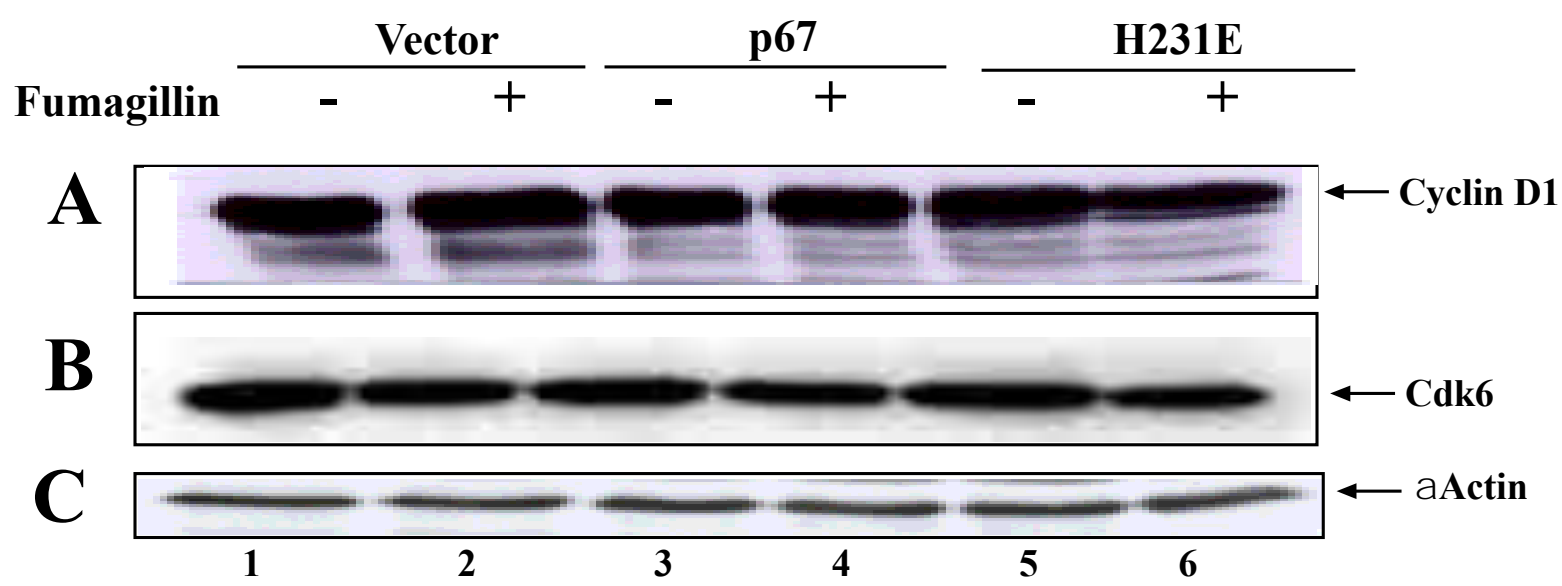

Fig6. Levels of cyclin D1 and Cdk6 in C2C12 myoblasts constitutively expressing rat p67 and its mutant H231E. Similar experiments in cell extracts from different cell lines as mentioned in legend to Figure 5 were performed except the analyses were done for the levels of cyclin D1 and Cdk6 using antibodies specific to these proteins.

Our examinations of the level of cyclin E and cyclin A partner Cdk2 showed that p67 can decrease its level possibly through degradation and its D251 residue and the N-terminal acidic residue-rich sequences but not N-terminal lysine residue-rich domains of p67 are involved in this process. The levels of Cdk1, the partner of cyclin A and cyclin B1 were mostly higher as the level of endogeneous p67 was increased by treating cells with fumagillin. P67 mutants like D251A, D6/2, and K1K2, which do not bind to wild type p67, have not much effect on this increment of Cdk1 level (Fig. 3B). Since p67 has significant impact in cycling B1 degradation (Fig. 3A) while increasing Cdk1 level, it is possible that p67 may be involved in $\mathrm{G}_{2} / \mathrm{M}$-phase transition and beyond. The levels of $\mathrm{Cdk} 4$ and Cdk6, the partners of cycling D1, which controls almost the entire $\mathrm{G}_{1}$-phase of the cell cycle, decreased slightly when p67's endogenous level was increased in rat p67-expressing cells or cells expressing p67-mutants D251, H231E, or K1K2. Therefore, p67 may be involved in the inhibition of cell cycle progression at the $\mathrm{G}_{1}$-phase by modulating the overall concentration of cycling D1/Cdk4 complex and/or cycling D1/Cdk6 complex.

All together, our data revealed the involvement of p67 in the cell cycle regulation by modulating several important steps. For example, p67 is involved in (i) the inhibition of cell cycle progression at 
the $\mathrm{G}_{1}$-phase; (ii) proteolysis processing to maturation of "pro" cycling $\mathrm{E}$ to its mature form, which regulates the transition of $\mathrm{G}_{1}$-phase into S-phase; (iii) proteolysis processing of "pro" cyclin A to its mature form and also upregulating its overall level, possibly to have positive impact in DNA synthesis; (iv) the degradation of $\mathrm{Cdk} 2$, the partner of both cyclin $\mathrm{E}$ and cyclin $\mathrm{A}$, and thus differentially modulating the $\mathrm{G}_{1} / \mathrm{S}$-phase transition and DNA synthesis phase; and (v) the $\mathrm{G}_{2} / \mathrm{M}$-phase transition and beyond through processing to maturation of "pro" cyclin B1 to its mature form and upregulating the level of Cdk1 possibly through direct or indirect binding with the cyclin B1/Cdk1 complex. Our literature search revealed no information regarding the presence of the "pro" forms of cyclins like pro-cyclin E, pro-cyclin A, and pro-cyclin B1 in the $\mathrm{C} 2 \mathrm{C} 12$ myoblasts. At present, we do not know how the pro-forms of these cyclins generate after translation of their mRNAs and how p67 is involved in the processing of these pro-forms into their mature full-length functional forms in $\mathrm{C} 2 \mathrm{C} 12$ myoblasts. Possibility exits that cyclins E, A, and B1 are synthesized as longer precursors and p67, through its intermolecular proteolysis activity, cleaves these precursors to generate mature forms of these cyclins. Since some Cdks are degraded while others did not, it is possible that some specific sequence motifs are involved in such proteolytic processing reactions with p67. Nonetheless, p67 is involved in the degradation of cyclins and some Cdks in general. Transition from one phase of the cell cycle to the next depends upon the degradation of cyclins and in some extends degradation of Cdks (16-17). Our data thus suggest that p67, which is a cytoplasmic protein (1), is involved in the regulation of cell cycle by regulating the levels of cyclin/Cdk complexes through efficient translation and/or proteolytic degradation of certain cyclins and Cdks in the cytoplasm.

\section{REFERENCES}

[1] Datta, B. (2015) Diversified roles of p67/MetAP2 as a regulator of cell growth and differentiation, in tumor suppression, and in obesity. Review, Curr. Topics Biochem. Res. 16, 41-52.

[2] Datta, B., Chakrabarti, D., Roy, A.L. and Gupta, N.K. (1988) Roles of a 67 kDa polypeptide in reversal of protein synthesis inhibition in heme-deficient reticulocyte lysate. Proc. Natl. Acad. Sci., USA, 85, 3324-3328.

[3] Ghosh, A., Datta, R., Majumdar, A., Bhattacharya, M., and Datta, B. (2006) The N-terminal lysine residue-rich domain II and the 340-430 amino acid segment of eukaryotic initiation factor 2-associated glycoprotein p67 are the binding sites for the $\gamma$-subunit of eIF2. Exp. Cell Res. 312, 3184-3203.

[4] Majumdar, A., Ghosh, A., Datta, S., Prudner, B., and Datta, B. (2010) P67/MetAP2 suppresses K-RasV12 mediated transformation of NIH3T3 mouse fibroblasts in culture and in athymic mice. Biochemistry 49, 10146-10157.

[5] Meloche, S., and Pouyssegur, J. (2007) The ERK1/2 mitogen-activated protein kinase pathway as a master regulator of the G1- to S-phase transition. Oncogene 26, 3227-3239.

[6] Datta, B., Ghosh, A., Majumdar, A., and Datta, R. (2007) Auto-proteolysis of rat p67 generates several peptide fragments: the N-terminal fragment, p26, is required for the protection of eIF2 $\alpha$ from phosphorylation. Biochemistry 46, 3465-3475.

[7] Datta, B. (2009) Roles of P67/MetAP2 as a tumor suppressor - a review. Biochim. Biophys. Acta 1796, 281-292.

[8] Liu, S., Widom, J., Kemp, C. W., Crews, C. M., and Clardy, J. (1998) Structure of human methionine aminopeptidase-2 complexed with fumagillin. Science 282, 1324-1327.

[9] Datta, B., Datta, R., Majumdar, A., and Balusu, R. (2004) Treatment of cells with the angiogenic inhibitor, fumagillin results in increased stability of eukaryotic initiation factor 2-associated glycoprotein, p67, and reduced phosphorylation of extra-cellular signal-regulated kinases 1 and 2. Biochemistry 43, 14821-14831.

[10] Deschenes-Simard, X., Kottakis, F., Meloche, S. and Ferbeyre, G. (2014) ERKs in cancer: Friends or Foes? Cancer Res. 74, 412-419.

[11] Torii, S., Yamamoto, T., Tsuchiya, Y., and Nishida, E. (2006) ERK MAP kinase in G cell cycle progression and cancer. Cancer Sci. 97, 697-702.

[12] Roberts, P. J. and Der, C. J. (2007) Targeting the Raf-MEK-ERK mitogen-activated protein kinase cascade for the treatment of cancer. Oncogene, 26, 3291-3310.

[13] Malumbres, M. (2014) Cyclin-dependent kinases. Genome Biol. 15, 122-131. 
[14] Lim, S., and Kaldis, P. (2013) Cdks, cyclins and CKIs: roles beyond cell cycle regulation. Development 140, 3079-3093.

[15] Duronio, R. J. and Xiong, Y. (2013) Signaling pathways that control cell proliferation. Cold Spring Harb Perspect Biol. 5, 1-12.

[16] Benanti, J. A. (2012) Coordination of cell growth and division by the ubiquitin-proteasome system. Semin. Cell Dev. Biol. 23, 492-498.

[17] Chang, W.-L., Yu, C.-C., Chen, C.-S., Guh, J.-H. (2015) Tubulin-binding agents down-regulate matrix metalloproteinase-2 and -9 in human hormone-refractory prostate cancer cells - A critical role of Cdk1 in mitotic entry. Biochem. Pharm. 94, 12-21.

[18] Ghosh, A., Tammali, R., Balusu, R., Datta, R., Chattopadhyay, A., Bhattacharya, M., and Datta, B. (2014) Oligomerization of the eukaryotic initiation factor 2-associated glycoprotein p67 requires N-terminal 1-107 amino acid residues. Intl. J. Appl. Biotech. Biochem. 4, 25-44.

[19] Datta, B., Datta, R., Majumdar, A., and Ghosh, A. (2005) The stability of eukaryotic initiation factor 2-associated glycoprotein, p67, increases during skeletal muscle differentiation and that inhibits the phosphorylation of extracellular signal-regulated kinases 1 and 2. Exp. Cell Res. 303, 174-182.

[20] Datta, B., and Datta, R. (2014) Phosphorylation of eukaryotic initiation factor $2 \alpha$ during diferentiation of mouse myoblasts into myotubes is mediated by an unknown kinase. Intl. J. Adv. Res. Chem. Sci. 1, 1-5.

[21] Datta, B. Min, W., Burma, S., and Lengyel, P. (1998) Increase in p202 expression during skeletal muscle differentiation: Inhibition of MyoD protein expression and activity by p202. Mol. Cell. Biol. 18, 1074-1083. 